\title{
Verifying Behavioural Specifications in CafeOBJ Environment
}

\author{
Akira Mori and Kokichi Futatsugi \\ Japan Advanced Institute of Science and Technology, Hokuriku \\ 1-1 Asahidai Tatsunokuchi Nomi Ishikawa, 923-1292, JAPAN \\ \{amori,kokichi\}@jaist.ac.jp
}

\begin{abstract}
In this paper, we present techniques for automated verification of behavioural specifications using hidden algebra. Two non-trivial examples, the Alternating Bit Protocol and a snooping cache coherence protocol, are presented with complete specification code and proof scores for CafeOBJ verification system. The refinement proof based on behavioural coinduction is given for the first example, and the coherence proof based on invariance is given for the second.
\end{abstract}

\section{Introduction}

The promise of formal methods has been heard for a long time, but with a few exceptions, they have not lived up to expectations. The shortcomings are due to:

- gap between specification and implementation, leading to the overspecification problem, and

- lack of unified logical systems that cover entire software design/development process, necessitating human intervention.

In other words, there have not been appropriate logical foundations for software specification. Overpopulation of specification languages has led to idiosyncrasy that is so difficult to see through. If formal methods are to have industrial impact on software productivity, they have to offer seamless integrated support throughout specification, verification, and transformation.

In this paper, we report on several techniques developed for behavioural specification based on hidden algebra to demonstrate its potential to be an industrial-strength specification method in the future. Hidden algebra was developed in an attempt to give a semantics for software engineering, and for the object paradigm in particular, supporting correctness proofs that are as simple and mechanical as possible [1. It distinguishes hidden sorts from visible sorts. As hidden sorts model states of the system, equations of hidden sorts need to be interpreted in a different manner. For this, hidden algebra formalises a notion of behavioural abstraction, by defining behavioural satisfaction of equations. An effective proof method for behavioural equation has been developed, called behavioural coinduction, and used for various refinement proofs. 
The development of hidden algebra is not an isolated event. There are many other proposed methods for behavioural specification, whose purpose is to characterise how systems behave instead of how they are implemented, but very few computer support systems exist. The CafeOBJ [2] system offers an integrated environment for specification and verification in the tradition of the OBJ languages, and is the only system that supports behavioural specification based on hidden algebra. As part of an effort to show the capability of behavioural specification, we report on some of the new features of CafeOBJ by means of two non-trivial examples, the Alternating Bit Protocol and a snooping cache coherence protocol. Specification code and correctness proof scores for CafeOBJ are presented for a complete explanation.

The organisation of the paper is as follows: Section 2 briefly summarises theoretical backgrounds of hidden algebra, Section 3 and 4 detail the specification and verification of the Alternating Bit Protocol and snooping cache coherence protocol respectively, and Section [5]concludes with comparisons with other approaches and future plans.

\section{Hidden Algebra}

This section presents basic definitions of hidden algebra. See [1, 3] for more details.

Hidden algebra distinguishes hidden state values from visible data values. Data values are defined as elements of a fixed (ordinary) algebra $D$ with signature $\Psi$ and sort set $V$ such that for each $d \in D_{v}$ with $v \in V$ there is some $\psi \in \Psi_{[], v}$ interpreted as $d$ in $D$. On the other hand, (hidden) state values are defined as elements of a special algebra with syntactic restrictions on its signature.

Definition 1. A hidden signature (over $(V, \Psi, D))$ is a triple $\left(H, \Sigma, \Sigma^{b}\right)$, where $H$ is a set of hidden sorts disjoint from $V, \Sigma$ is an $(H \cup V)$-sorted signature with $\Psi \subseteq \Sigma$, and $\Sigma^{b} \subseteq \Sigma$ is a set of behavioural operations disjoint from $\Psi$, such that

- each $\sigma \in \Sigma_{w, s}$ with $w \in V^{*}$ and $s \in V$ lies in $\Psi_{w, s}$, and,

- each $\sigma \in \Sigma_{w, s}^{b}$ has exactly one element of $H$ in $w$.

$\left(H, \Sigma, \Sigma^{b}\right)$ may be abbreviated to $\Sigma$ leaving $\Sigma^{b}$ implicit. An operation $\sigma$ in $\Sigma_{w, s}^{b}$ is called a method if $s \in H$ and an attribute if $s \in V . \sigma \in \Sigma_{w, s}$ is called a hidden constant if $w \in V^{*}$ and $s \in H$.

Note that operations in $\Sigma_{w, s}-\Sigma_{w, s}^{b}$ may have more than one elements of $H$ in $w$. These non-behavioural operations were not considered in the original definition of hidden algebra [1. They have been introduced to increase expressiveness of hidden algebra [4, 5]. The above definition is due to [4] and is supported in the current CafeOBJ system.

Definition 2. Given a hidden signature $\left(H, \Sigma, \Sigma^{b}\right)$, a hidden $\Sigma$-algebra $A$ is a (many sorted) $\Sigma$-algebra $A$ such that $\left.A\right|_{\Psi}=D$. 
As mentioned above, the elements of $A_{v}$ where $v \in V$ is thought of as data values and the elements of $A_{h}$ where $h \in H$ as state values.

Definition 3. A hidden (or behavioural) theory (or specification) is a quadruple $\left(H, \Sigma, \Sigma^{b}, E\right)$, where $\left(H, \Sigma, \Sigma^{b}\right)$ is a hidden signature and $E$ is a set of $\Sigma$-(conditional) equations; we may write $(\Sigma, E)$ for short.

Example 1. We present a behavioural specification of a flag object using CafeOBJ notations [3]. A flag object is either up or down, and there are methods to put it up, to put it down, and to reverse its state:

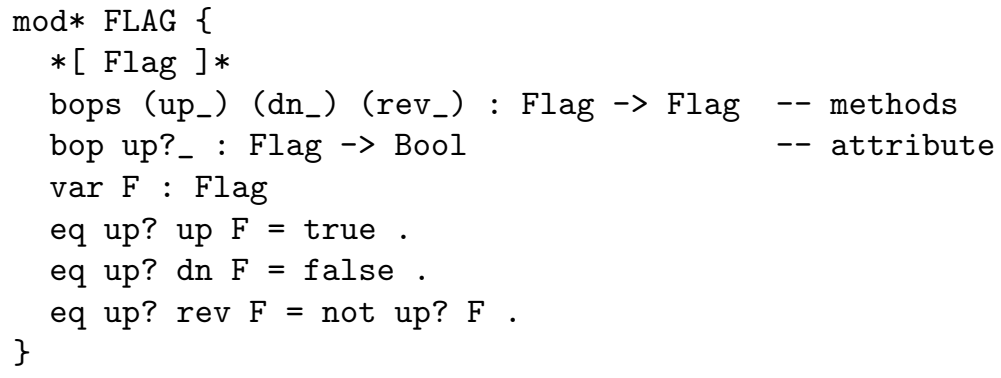

A CafeOBJ keyword mod* means that the module has a loose behavioural semantics in contrast with a tight (initial algebra) semantics specified by the keyword mod!. A pair of starred brackets $*[\ldots] *$ is used for sort declaration and $*$ indicates that the declared sort is a hidden sort. For visible sorts, [ . . ] is used. The keyword bop declares behavioural operations in $\Sigma^{b}$ (i.e., attributes and methods).

The meaning of the FLAG specification should be clear, however, some of the intended behaviours of the flag object, for example, a behavioural equation rev rev $F=F$ cannot be deduced from the FLAG specification using ordinary equational reasoning 1. This means that ordinary satisfaction of equations is too strict for behavioural equations and a weaker notion of satisfaction based on indistinguishability ${ }^{2}$ is needed. In hidden algebra, it is formalised using contexts as follows.

Definition 4. Given a hidden signature $\left(H, \Sigma, \Sigma^{b}\right)$, a behavioural context is a term having a single occurrence of a special variable of hidden-sort denoted by $z$ and is formed by the following rules:

- any variable $z$ (of any sort) is a behavioural context,

- for any $\sigma \in \Sigma_{v h, s}^{b}$, any behavioural context $c$ of sort $h$, and any tuple of ground terms $t \in\left(T_{\Sigma}\right)_{v}, \sigma(t, c)$ is a behavioural context, where $v \in V^{*}$ and $h \in H$. If $s \in V$, then $\sigma(t, c)$ is called visible, otherwise it is called hidden.

\footnotetext{
${ }^{1}$ Not to mention induction.

${ }^{2}$ By means of method application and attribute observation.
} 
Given a hidden $\Sigma$-algebra $A$, two elements $a$ and $a^{\prime}$ of the same carrier set $A_{s}$ are said to be behaviourally equivalent, denoted by $a \sim_{s} a^{\prime}$ (or just $a \sim a^{\prime}$ ) iff $A_{c}(a)=A_{c}\left(a^{\prime}\right)$ for all visible behavioural contexts $c$, where $A_{c}$ denotes the function interpreting the context $c$ as an operation on $A$.

$A$ hidden $\Sigma$-algebra A behaviourally satisfies a (conditional) equation $e$ of the form $(\forall X) t=t^{\prime}$ if $t_{1}=t_{1}^{\prime}, \ldots, t_{m}=t_{m}^{\prime}$ iff

$$
\theta^{*}(t) \sim \theta^{*}\left(t^{\prime}\right) \text { whenever } \theta^{*}\left(t_{j}\right) \sim \theta^{*}\left(t_{j}^{\prime}\right) \text { for all } j=1, \ldots, m
$$

for every valuation $\theta: X \rightarrow A$. In this case, we write $A \equiv_{\Sigma}$ e. We may drop the subscript $\Sigma$.

Note that for visible sorted equations, there is no difference between ordinary satisfaction and behavioural satisfaction. We will use the symbol $\sim$ instead of $=$ when the equation should be interpreted by behavioural satisfaction. Such equations are called behavioural equations and specified by keywords beq, bceq in CafeOBJ, instead of eq, ceq where ceq stands for conditional equations.

The first effective algebraic proof technique for behavioural equivalence was context induction [6], however, a more comprehensive technique based on maximality has been developed.

Definition 5. Given a hidden signature $\Sigma$, and a hidden $\Sigma$-algebra A, behavioural congruence on $A$ is a $\Sigma^{b}$-congruence which is identity on visible sorts.

Theorem 1. Given a hidden signature $\Sigma$ and a hidden $\Sigma$-algebra $A$, then behavioural equivalence is the largest behavioural congruence on $A$.

See [1, 4, 5] for the proof.

Thanks to this theorem, one can show $a \sim a^{\prime}$ by finding some behavioural congruence that relates $a$ and $a^{\prime} 3$. This is what is called behavioural (or hidden) coinduction and justifies a variety of techniques for proving behavioural equivalence. For example, to show that every FLAG-algebra satisfies the equation $(\forall \mathrm{F}: \mathrm{Flag}$ ) rev rev $\mathrm{F}=\mathrm{F}$, one only needs to show that up? rev rev $\mathrm{F}=$ up? $\mathrm{F}$. This is a special case of attribute coherent theory, where the equivalence on attributes is behavioural equivalence 4. CafeOBJ system automatically checks if this happens every time a new module is loaded. When this is the case, showing behavioural satisfaction is automatic.

Non-behavioural operations have been introduced to enhance the expressive power of hidden algebra [4], for example for modular construction of behavioural specifications. However, non-behavioural constructors may not preserve behavioural equivalence and therefore may ruin the soundness of equational reasoning (specifically the congruence rule substituting equal with equal). The next definition gives a sufficient condition for sound equational deduction.

\footnotetext{
${ }^{3}$ Note that the situation is very much similar to the technique in process algebra for demonstrating (strong) bisimilarity through bisimulation [7.

${ }^{4}$ Visit the website at UCSD, http://www.cs.ucsd.edu/groups/links.
} 
Definition 6. Given a hidden signature $\Sigma$ and a hidden $\Sigma$-algebra $A$, an operation $\sigma \in \Sigma_{w, s}-\Sigma_{w, s}^{b}$ is said to be behaviourally coherent iff it preserves the behavioural equivalence on $A$, that is, $A_{\sigma}\left(a_{1}, a_{2}, \ldots, a_{n}\right) \sim_{s} A_{\sigma}\left(a_{1}^{\prime}, a_{2}^{\prime}, \ldots, a_{n}^{\prime}\right)$ if $a_{1} \sim_{s_{1}} a_{1}^{\prime}, a_{2} \sim_{s_{2}} a_{2}^{\prime}, \ldots, a_{n} \sim_{s_{n}} a_{n}^{\prime}$ for all $\left(a_{1}, a_{2}, \ldots, a_{n}\right)$ and $\left(a_{1}^{\prime}, a_{2}^{\prime}, \ldots, a_{n}^{\prime}\right)$ in $A_{s_{1}} \times A_{s_{2}} \times \cdots \times A_{s_{n}}$, where $w=s_{1} s_{2} \cdots s_{n}$.

The next theorem is due to [4. See also 5] for another proof (in a slightly different setting) and examples.

Theorem 2. If all operations in $\Sigma-\Sigma^{b}$ are behaviourally coherent, then ordinary equational deduction is sound for behavioural equations.

\section{The Alternating Bit Protocol}

Now that we have introduced all necessary concepts, let us turn to some interesting specifications and their verification.

The first example is the classic Alternating Bit Protocol (ABP) 8]. The protocol is designed to achieve secure communication through unreliable channels that may lose or duplicate packets. The model of the protocol consists of four agents, the sender, the receiver, the message (msg) channel from the sender to the receiver, and the acknowledgement (ack) channel from the receiver to the sender.

msg

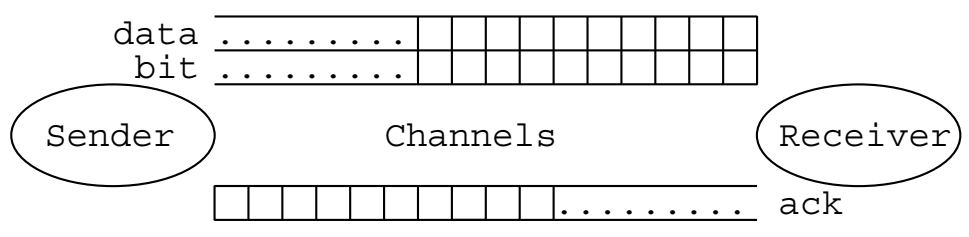

ack

Fig. 1. The Alternating Bit Protocol

In addition to data that are transmitted, the protocol uses extra bits to recover potential errors (loss and duplication) in the channels. The sender sends messages, each of which consists of a data and a bit, along the msg channel, and receives acknowledgement bits from the ack channel. The receiver receives messages from the msg channel and sends back acknowledgements along the ack channel. The channel is modeled as a simple unbounded queue that may lose or duplicate packets, but does not alter the order of packets. As its name suggests, the key trick of the protocol is the bit alternation that takes place when a packet having the right bit is received. This is a good example for formal 
specification and verification methods, since its procedure is extremely simple, yet very sophisticated.

We model the protocol in terms of actions taken by the sender and the receiver. Each agent maintains three types of information, a data, a bit, and a timer. Each checks the incoming bit with the bit it has and then determines the next action. We have included the timer since it is necessary to prevent deadlocks.

\section{Sender:}

- receives a right ack (the same bit as it has) from the ack channel, accepts and keeps a new data, alternates the bit, sends a couple of the new data and the new bit along the msg channel, sets a timer;

- receives a wrong ack (does nothing);

- resends a couple of the current data and bit when the timer goes off.

\section{Receiver:}

- receives a message with a right bit (the opposite bit of the one it has) from the msg channel, keeps the new data (for future delivery), alternates the bit, sends the new bit along the ack channel, sets a timer;

- receives a message with a wrong bit (does nothing);

- resends the current bit when the timer goes off.

Below is a more or less faithful behavioural specification of the descriptions above.

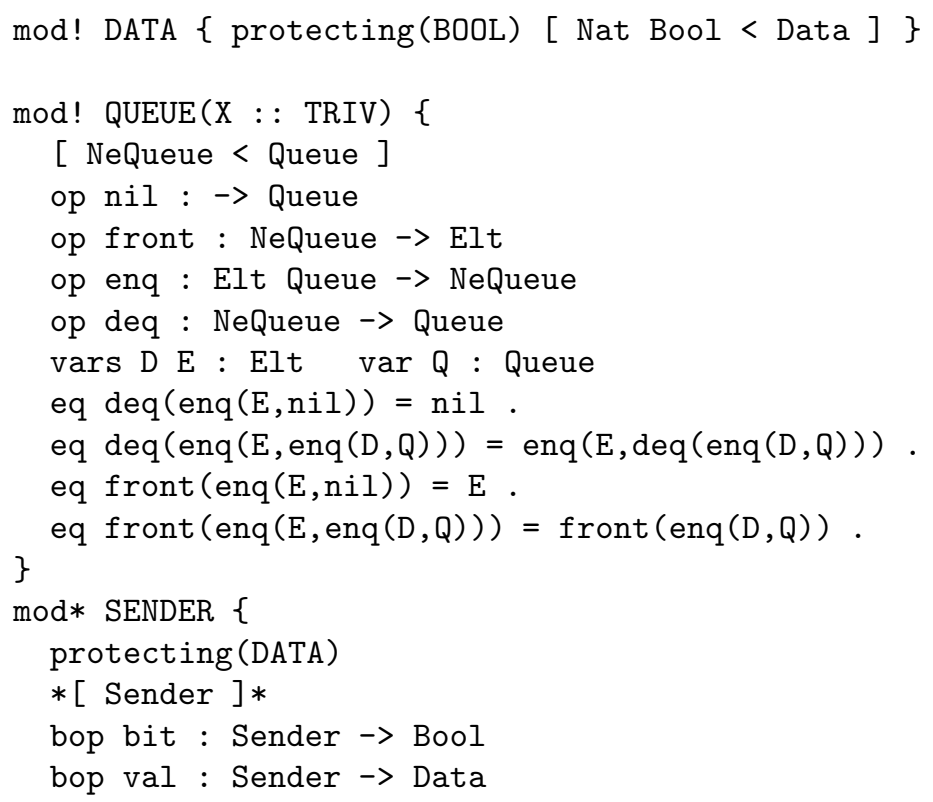

${ }^{5}$ However, the timer does not appear in the behavioural specification. 


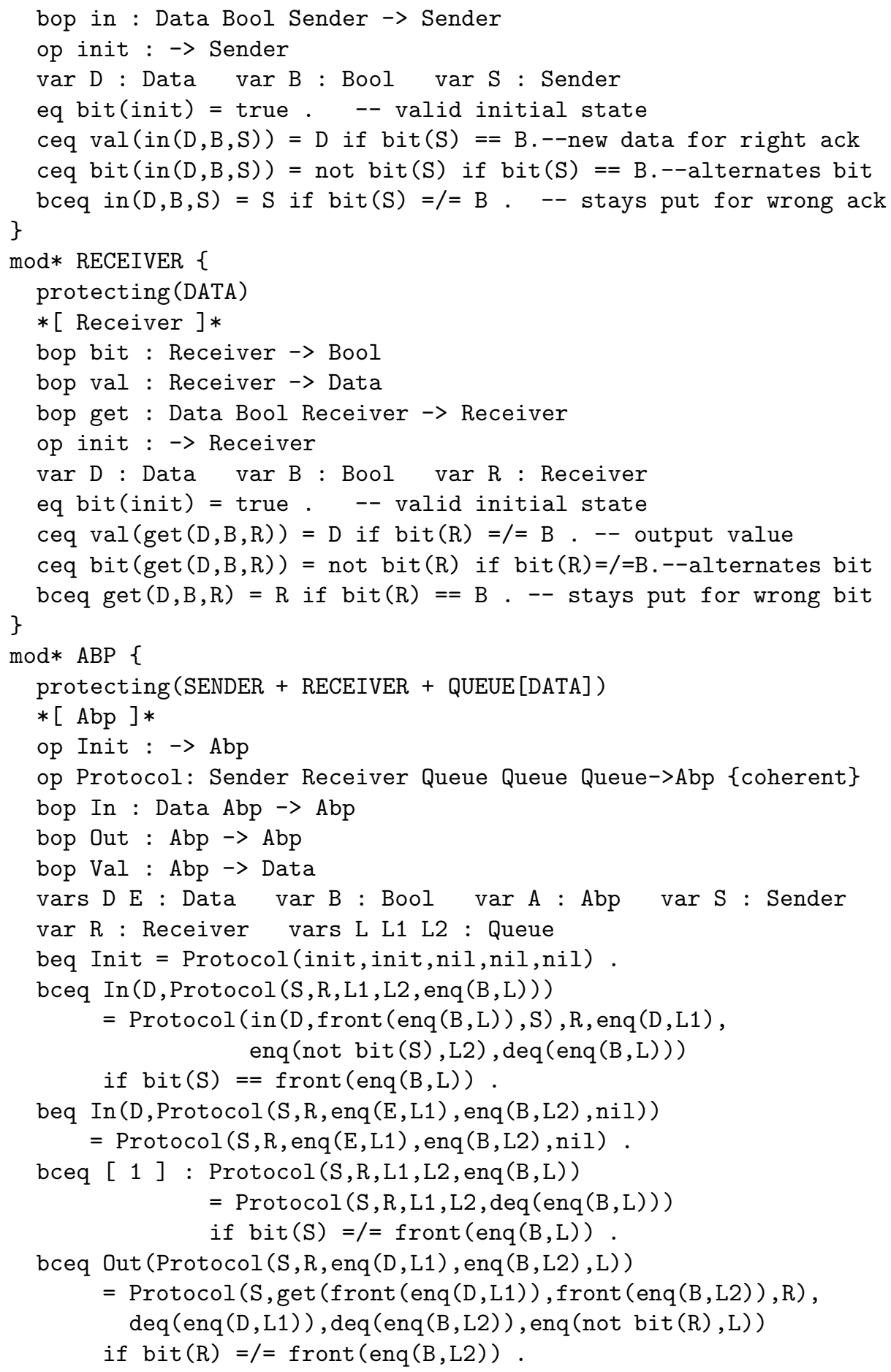




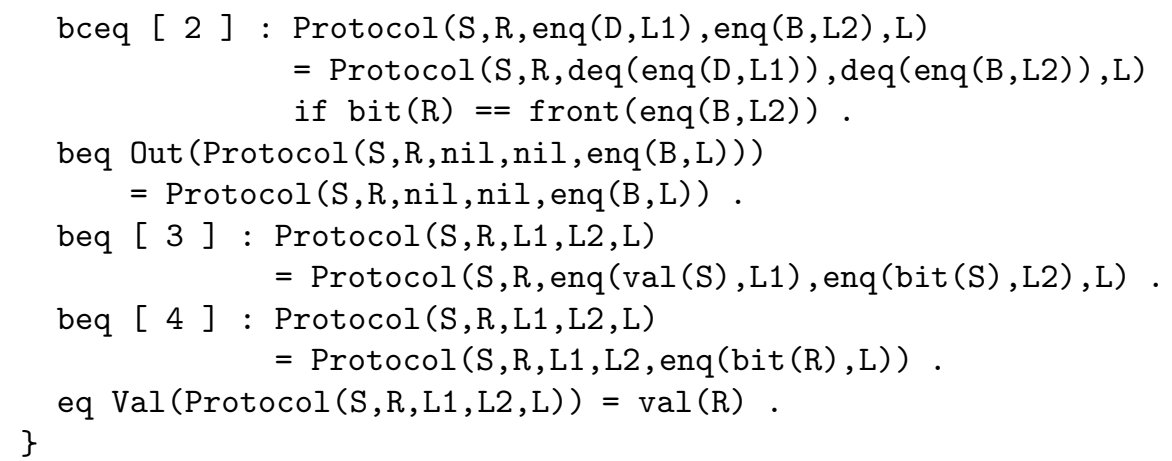

A few remarks about the specification are in order.

- Transmitted data and alternation bits are specified using CafeOBJ's default built-in modules Nat (the natural numbers) and Bool (the Booleans).

- A parameterised module QUEUE and subsorts Nat and Bool are used to avoid multiple presence of QUEUE modules. Also subsort NeQueue is used to avoid introducing user-defined error elements.

- Modules are imported by protecting declaration. It instructs that the semantics of imported modules should not be altered. There are other importation modes than protecting. See 3 for details and semantics.

- Initial states are defined as hidden constants. Attribute values for initial states can be defined by visible equations. The initial state of ABP may look strange since the data transmission can only be started by the Receiver taking a time-out action 6 . This is rather a syntactic convenience, i.e., one could have put an appropriate acknowledgement bit in the ack channel.

- Module expressions such as SENDER + RECEIVER + QUEUE [DATA] avoid multiple internal copies of shared modules.

- A non-behavioural operation Protocol is declared coherent in the ABP module. Thus one can freely use equational reasoning through reduction commands reduce or red in CafeOBJ. The coherence of Protocol needs to be proved separately, however, it is trivial and omitted here. One can think of coherent operations as behavioural constructors just like ordinary data type constructors. They are used to define legitimate system configurations in terms of behavioural sub-modules.

CafeOBJ keywords appearing in the specification should be easily interpreted by corresponding hidden algebra notions. Interested readers are referred to 3 . for CafeOBJ specific details.

Interpreting equations [ 1 ], [ 2 ], [ 3 ], and [ 4 ] with (ordinary) strict satisfaction of equations will not make sense. For example, one might think that equations [ 3 ] and [ 4 ] bring about inconsistency since the operation Protocol acts as a constructor. One has to remember, however, that they are behavioural equations that only need to be satisfied behaviourally. By virtue of

${ }^{6}$ Not by the Sender. 
behavioural satisfaction, equations [ 1 ] and [ 2 ] state that wrong packets are removed without making any trace while [ 3 ] and [ 4 ] state that timeout events are invisible and resending actions should not affect the following behaviour of the protocol. Writing behavioural equations always runs a risk of producing inconsistent specifications since they imply infinite number of visible equations. This is probably the most difficult aspect of behavioural specification.

We define the notion of behavioural refinement first.

Definition 7. A hidden signature map $\varphi:(H, \Sigma) \rightarrow\left(H^{\prime}, \Sigma^{\prime}\right)$ is a signature morphism $\varphi: \Sigma \rightarrow \Sigma^{\prime}$ that preserves hidden sorts and behavioural operations, and that is the identity on $(V, \Psi)$. A hidden signature map $\varphi:(\Sigma, E) \rightarrow\left(\Sigma^{\prime}, E^{\prime}\right)$ is a refinement iff for every $\left(\Sigma^{\prime}, E^{\prime}\right)$-algebra $M^{\prime}$ we have $\varphi M^{\prime} \equiv_{\Sigma} E .\left(\varphi M^{\prime}\right.$ denotes $M^{\prime}$ viewed as a $\Sigma$-algebra.)

It can be shown that $\varphi$ is a refinement iff all visible consequences of the abstract specification hold in the concrete specification [9]:

Proposition 1. A hidden signature $\operatorname{map} \varphi:(\Sigma, E) \rightarrow\left(\Sigma^{\prime}, E^{\prime}\right)$ is a refinement iff $E^{\prime} \models \varphi(c[e])$ for each $e \in E$ and each visible $\Sigma$-context $c$, where if $e$ is the equation $(\forall X) t=t^{\prime}$, then $c[e]$ denotes the equation $(\forall X) c[t]=c\left[t^{\prime}\right]$.

Now we want to show that ABP is a refinement of the following behavioural specification BUF of a buffer of capacity one.

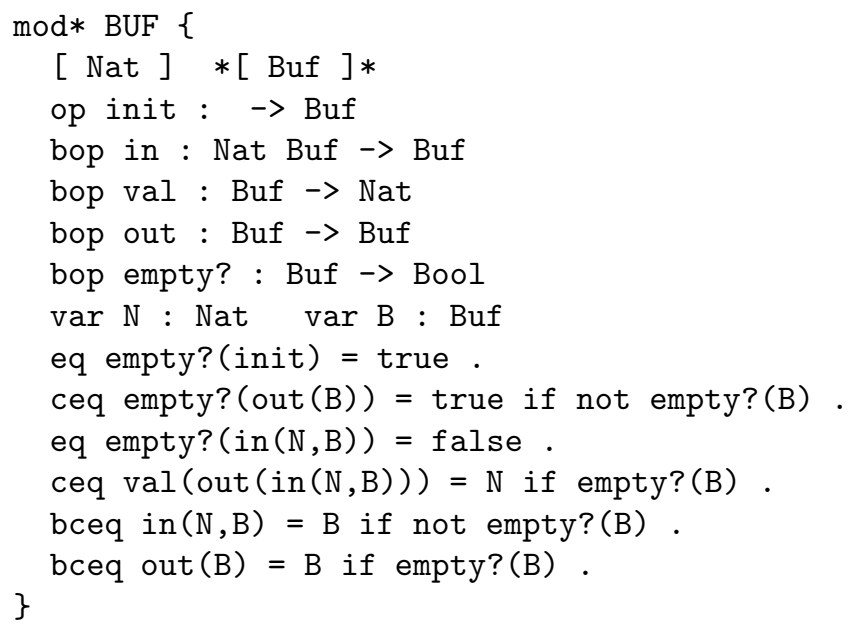

The meaning of this specification should be clear, however, note that the output value is secure only after the out operation.

In order to demonstrate 7 refinement from BUF to ABP, we first need to find an underlying signature map. For this, we define an ABP counterpart Empty? of empty? as follows.

\footnotetext{
${ }^{7}$ Before starting formal verification, one can check how ABP works via symbolic execution. See Appendix $\mathrm{A}$ for a CafeOBJ session of simple ABP reductions.
} 
bop Empty? : Abp $\rightarrow$ Bool .

var S : Sender var R : Receiver vars L L1 L2 : Queue

eq Empty? (Protocol $(S, R, L 1, L 2, L))=\operatorname{bit}(S)==\operatorname{bit}(R)$.

Then the refinement proof requires us to show all equations from BUF are valid in ABP as well. This is done by finding an appropriate candidate relation $R$ on $\mathrm{Abp}$ and showing that it is a behavioural congruence. In general, this is highly non-trivial. However, a closer look at behavioural equations [ 1 ] - [ 4 ] of $\mathrm{ABP}$ leads to the observation that for any reachable state of the protocol there is a behaviourally equivalent state whose channels are all empty. This is the ingenious aspect of the protocol that achieves robust communication against loss and duplication in the channels.

We are going to make this observation precise by creating CafeOBJ scores. However, since CafeOBJ does not have full theorem proving capability (only reductions), we need some maneuvers for quantified variables and conditionals, and also for rewriting directions and orders. The CafeOBJ commands for controlling these are omitted from the following proof score.

We define the candidate relation $R$ to be

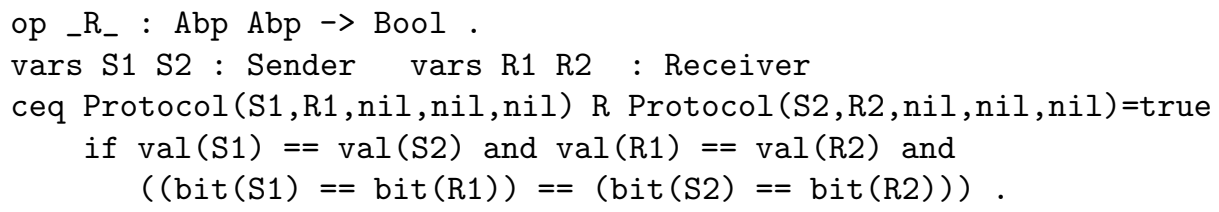

It suffices to define $R$ on states with empty channels since behavioural equations in $\mathrm{ABP}$ are closed within reachable states Protocol (S, R, L1, L2, L) where

$$
\mathrm{L} 2=a^{*} b^{*}, \mathrm{~L}=b^{*} \bar{a}^{*}, a=\operatorname{bit}(\mathrm{S}), b=\operatorname{bit}(\mathrm{R}) .
$$

(The head of the queue is placed to the right, and $\bar{a}$ stands for the opposite bit of $a$.) It is very interesting to note that only one bit change occurs in the concatenation of $L 2$ and $L$, depending upon whether $a=b$ or $a \neq b$. The $a=b$ case corresponds to the empty state ready to accept a new data, and $a \neq b$ to the full state having an accepted data waiting to be delivered. Identifying these two groups of states is essential for any attempt at formal verification of the ABP. It is also noted that the use of a coherent non-behavioural operation (that is, Protocol) simplifies the specification together with the enabled power of equational reasoning.

Now to check congruence of $R$.

-- universally quantified variables

-- i.e., Theorem of (Hidden) Constants

ops s s1 s2 s3 : -> Sender. ops r r1 r2 r3 : -> Receiver.

ops a a1 a2 a3 : -> Abp. op e : -> Nat.

-- a matches a2 while a1 matches a3,

eq $\mathrm{a}=\operatorname{Protocol}(\mathrm{s}, \mathrm{r}, \mathrm{nil}, \mathrm{nil}, \mathrm{nil})$. 


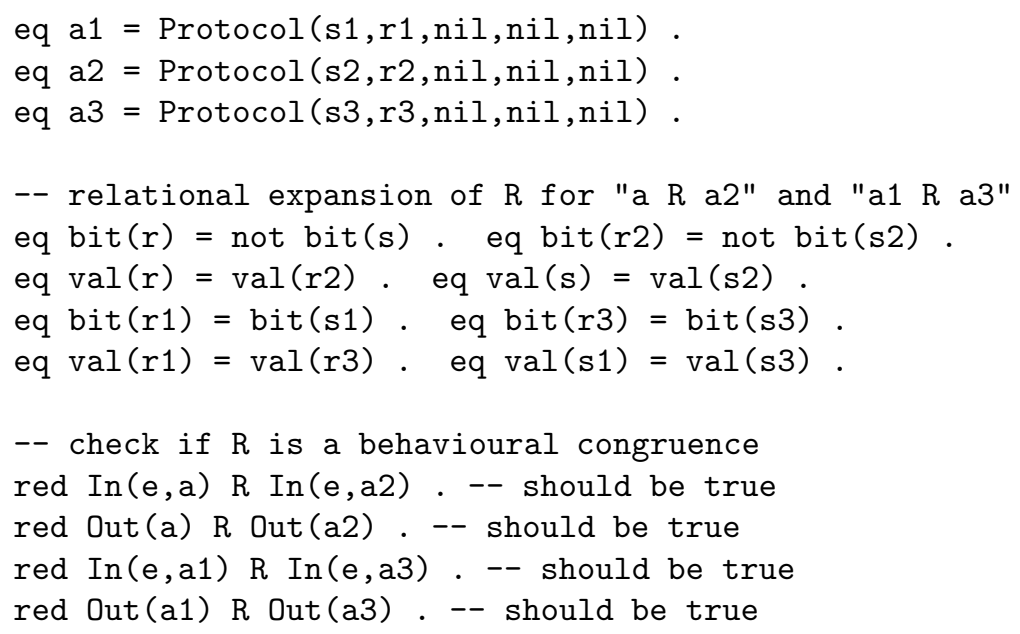

CafeOBJ gives true for all reductions.

We have used the Theorem of (Hidden) Constants to perform proof rules for universal quantifiers by introducing fresh constant operations. The soundness of the method is proved in [1,5]. We have also used implication elimination and case analysis. One can mimic proof rules of first order logic with equality in a similar manner. An automated proof support tool called Kumo has been developed for OBJ3 and CafeOBJ systems [10. Kumo is not just a proof checking tool, but also helps to publish proofs over the internet, i.e., Kumo creates webpages for proofs as it checks proofs given in the proof scripts.

Now that we have established a behavioural congruence, we can use behavioural coinduction to prove behavioural equations. The general case is rather complicated, however, if we restrict attention to ABP states with empty channels as the representatives of equivalent classes modulo $R$, the coinduction proof becomes manageable using reduction. The following CafeOBJ score does this by unfolding conditionals. Note that the constants a and a1 are the same as defined earlier.

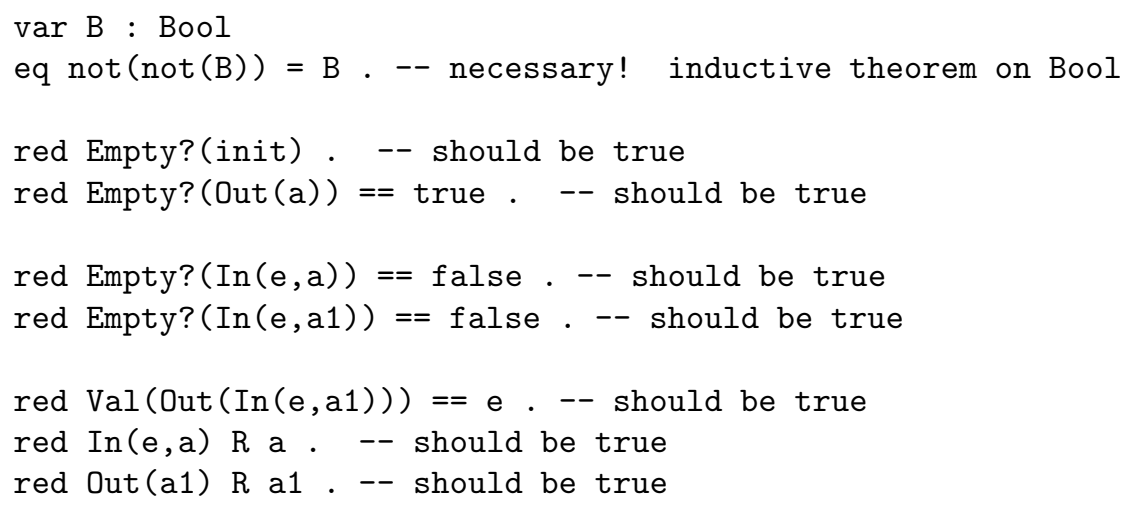


Again, CafeOBJ returns true for all reductions 8. We can now claim that

"ABP implements BUF when there are no errors in channels."

However, the error recovering capability of ABP is automatic since any loss or duplication in the channel does not affect the (now proved) behavioural congruence of $R$. Therefore, we arrive at the following correctness theorem for ABP.

Theorem 3. As far as the reachable states are concerned, errors in channels do not affect the behaviour of the protocol. In other words, the ABP is a robust implementation of a buffer BUF of capacity one.

Mechanising the whole process should be possible by defining an appropriate merge operation on queues and the filter that checks bit changes. However, it is going to be an induction flood. We do not feel it necessary to completely mechanise proofs when human can do a much better job. We will see a fully automated verification example using a theorem prover in the next section.

\section{Cache Coherence Protocol}

In this section, we present a somewhat more implementation-oriented example of a cache coherence protocol.

A number of computation units share a main memory through a common access bus. Each unit issues access requests through the bus and maintains its own cached copy. In order to assure consistency among cached copies, the protocol watches (snoops) requests on the bus and takes appropriate actions depending on the flag values that tracks cache states. A coherence condition we consider is the following.

"If there are two shared copies, they must be of the same value."

We model the Illinois cache protocol [11] following [12. There are three types of requests, (read, write, and replacement), and four different flag values (cache states):

- invalid - obsolete;

- shared - not modified, possible copies in other caches;

- valid-exclusive - not modified, only copy in caches;

- dirty - modified, only copy in caches.

Coherence actions are taken as follows, depending on the cache state of the requesting unit and the existence of other cached copies.

Read Hit. the cache (in the requesting unit) is not invalid, no extra action; Read Miss. the cache is invalid, look for other cached copies;

${ }^{8}$ The double negation equation is necessary. The situation is frequently encountered, in which coinduction (or invariance proof) on hidden sorts requires induction on visible sorts. 


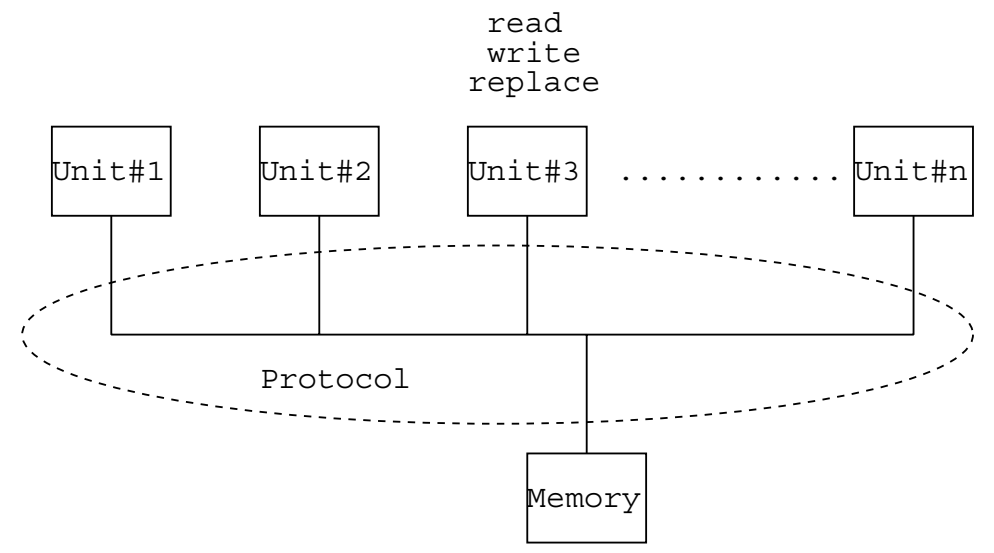

Fig. 2. The Illinois Cache Protocol

- dirty cache provides the latest value, both end up shared;

- any other valid-exclusive or shared cache provides the latest value, all caches having a copy end up shard;

- no cached copy, get a valid-exclusive copy from the main memory;

Write Hit. the cache is not invalid;

- the cache is dirty, no extra action;

- valid-exclusive turns dirty;

- shared turns dirty, all other shared copies are invalid-ated;

Write Miss. invalid turns dirty, all cached copies are invalid-ated;

Replacement. dirty copy is written back to the main memory, stays dirty;

Below is a behavioural specification PROTOCOL of the above procedure.

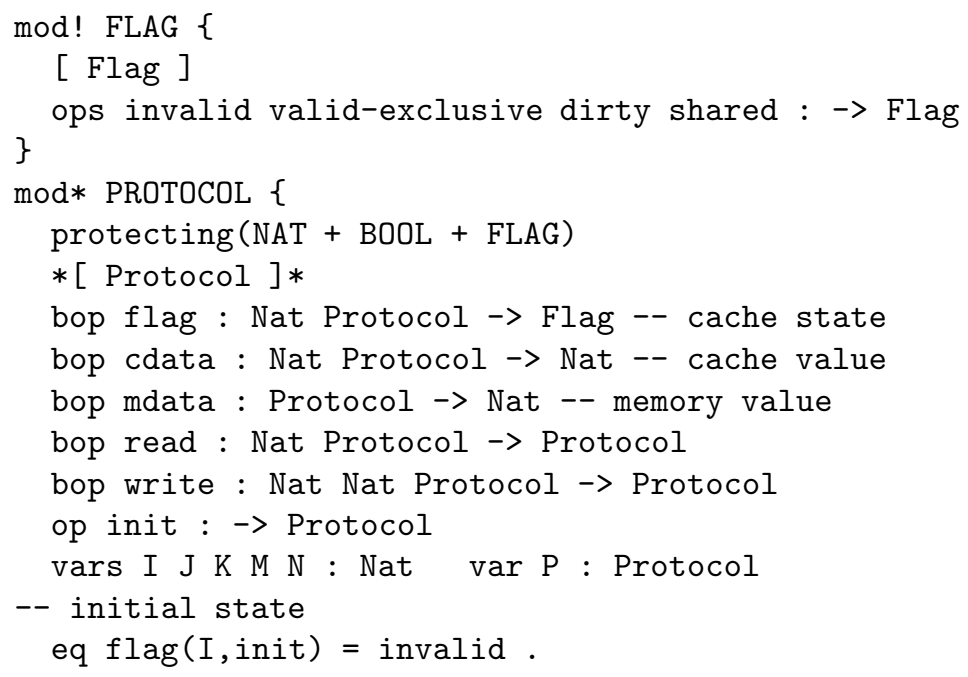




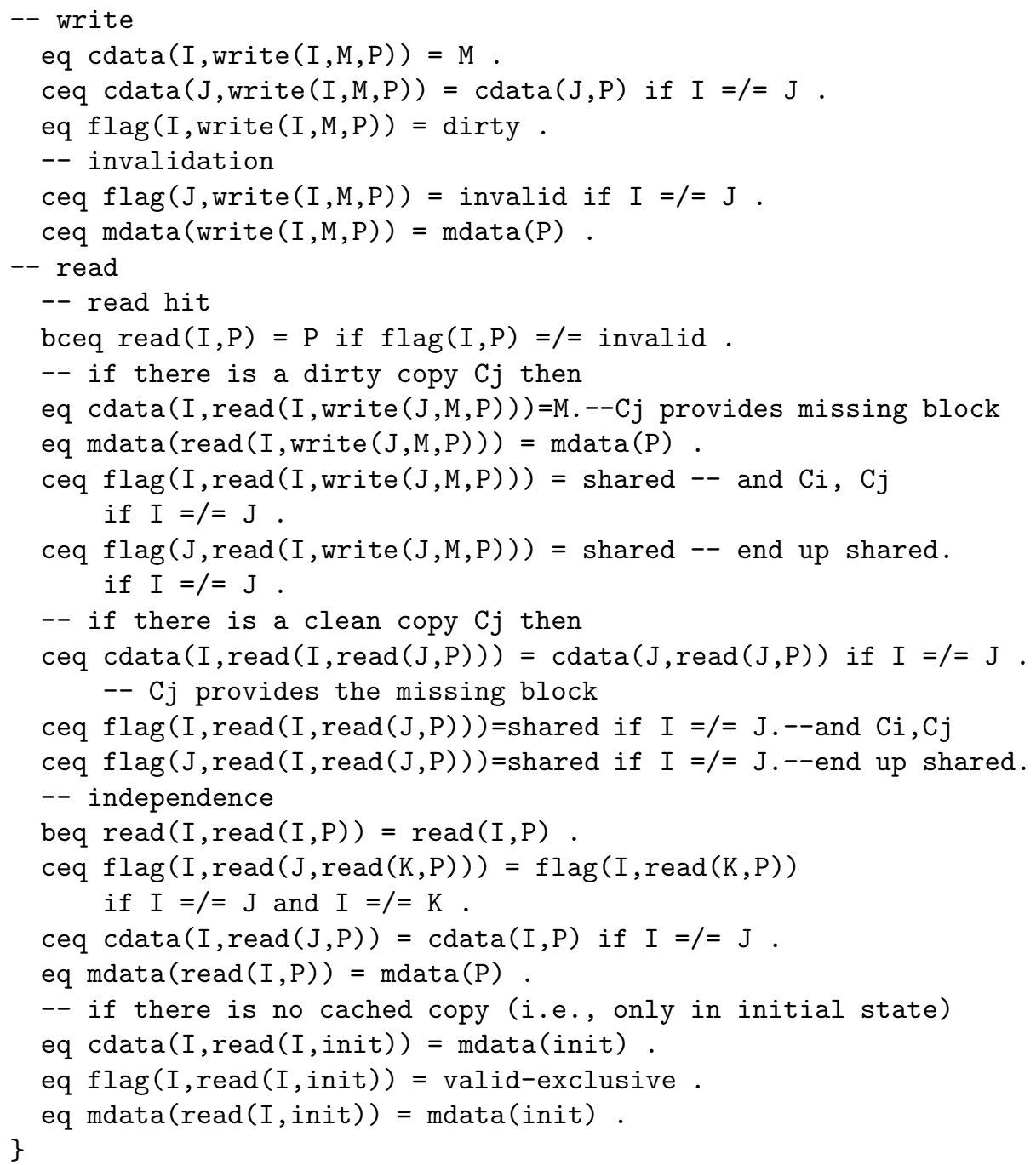

A few remarks on the specification.

- It does not have any non-behavioural operations, thus equational reasoning is trivially sound.

- Unlike ABP, it handles arbitrary number of units, which is modeled by operations having an index argument for the unit.

- It only models a single memory block since multiple blocks can be modeled by an extra argument of each operation.

- It does not model replacement since it has little effect on the coherence property (and due to the limit of space).

- It is written in a deterministic fashion to allow symbolic execution with term rewriting (reduction) in CafeOBJ. It is based on the following decision and observation. 
- The unit that made a read request most recently is the one who provides the value.

- write always gets a dirty copy and invalidates other copies.

As we mentioned earlier, getting inconsistent specifications is much more likely in behavioural specification. We had great help from a resolution / paramodulation theorem prover that was implemented on CafeOBJ, in finding subtle inconsistencies among attribute values in the earlier versions.

Now we want to show the following coherence condition.

In all reachable states $\mathrm{S}:$ Protocol,

$\forall \mathrm{I}, \mathrm{J}: \operatorname{Nat} \mathrm{flag}(\mathrm{I}, \mathrm{S})=\mathrm{flag}(\mathrm{J}, \mathrm{S})=\operatorname{shared} \Rightarrow \operatorname{cdata}(\mathrm{I}, \mathrm{S})=\operatorname{cdata}(\mathrm{J}, \mathrm{S})$.

Equational reasoning cannot prove this because it cannot trace all reachable states with respect to behavioural equivalence. The proof requires a technique similar to coinduction, known as invariance proof. (See [13] for a categorytheoretic characterisation of invariants including relation to bisimulation, and [14] for a fixed-point characterisation.) It is a well-studied topic in the field of program verification and is also a prototype of (safety) model checking. There are two equivalent ways of proving invariance, forward and backward. Backward calculation of largest invariants (also known as weakest preconditions) is particularly suitable for behavioural specification. Making a long story short, to prove that a predicate $P(S: h)$ on a hidden sort $h$ is an invariant for all reachable states, it suffices to show that the initial state satisfies $P$ and that $P$ is closed under all methods, that is, $\forall S: h \forall X: v P(S) \Rightarrow P(m(X, S))$ for all method $m \in \Sigma_{v h, h}^{b}$ (e.g., [13]). In our case, the predicate on Protocol is defined as follows.

\section{$P(S: P r o t o c o l)=$$$
\forall \mathrm{I}, \mathrm{J}: \operatorname{Nat} \mathrm{flag}(\mathrm{I}, \mathrm{S})=\mathrm{flag}(\mathrm{J}, \mathrm{S})=\operatorname{shared} \Rightarrow \operatorname{cdata}(\mathrm{I}, \mathrm{S})=\operatorname{cdata}(J, S) \text {. }
$$

Thus, in order to prove the invariance of $P$, we have to prove the following formulas.

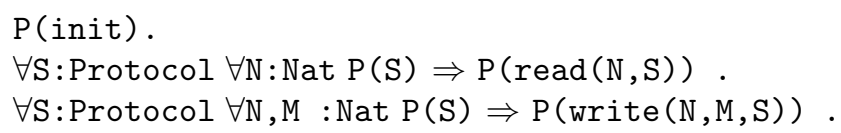

It took about five minutes for our resolution/paramodulation theorem prover to get the proofs of these on a Pentium $266 \mathrm{MHz}$ PC 9. The process is completely automatic except for the inductive theorems on visible sort (in our case, Flag) mentioned below. The user gives a predicate and the system generates all proof obligations in clausal form, which are then passed to the prover. Taking five minutes may not sound great. However, the proof is not easy for a human either. One of the authors tried the proof and it took more than five minutes. It is a straight-forward case analysis and we believe that resolution is particularly

${ }^{9}$ The first one is trivial, the third one is easy, and the second one took almost the entire execution time. 
useful for this type of theorem proving. Although this proof is probably close to the practical limit of automated theorem provers, we see many interesting applications to behavioural specifications.

For example, the above proof requires inductive theorems on the FLAG specification 10 .

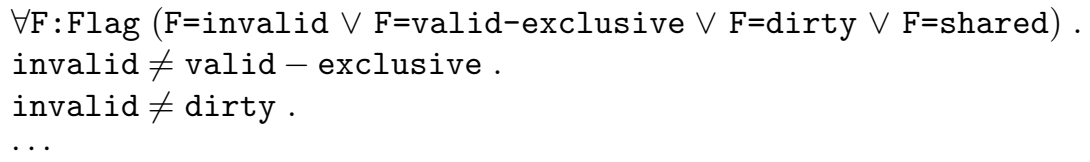

It is an interesting challenge to work with full-fledged inductive theorem provers so that these inductive theorems are discovered automatically when needed.

The example here may be interesting as a cache coherence proof in its own right since there are very few examples that are: 1) fully automated (no human intervention), 2) ready for implementation , 3) free of syntactic translation, and 4) machine supported, despite the large volume of research devoted to the topic.

The invariance proof is too simple when regarded as model checking. However, by combining iterative calculations of largest fixed-points, one can perform full-scale model checking. We are currently working on a so-called behavioural model checker that manages predicate representations, obligation generation, and theorem prover (resolution + induction) control. This is an interesting area of study, i.e., the combination of deductive and model-theoretic methods for software verification.

\section{Concluding Remarks}

In this paper, we have presented methods for verifying behavioural specifications based on hidden algebra. We have reported on CafeOBJ's automation support with a couple of non-trivial examples, the Alternating Bit Protocol and a snooping cache coherence protocol. The refinement proof based on behavioural coinduction is given for the first example, and the coherence proof based on invariance is given for the second. We have included almost complete specification code and proof scores since they are much more valuable and effective sometimes than verbal descriptions and explanations.

Since the hidden algebra (or behavioural) approach is a relatively new branch of formal methods, we compare its characteristics with other well-established 11 methods.

Process algebra treats observability based on events (rather than values) and is very suitable for defining process interaction and evolution, which is one of the unexplored fields of study in hidden algebra. On the other hand, hidden algebra fits very well the notion of object orientation (methods/ attributes, encapsulated states, inheritance, overloading, and so on). The

\footnotetext{
${ }_{10}$ These were given to the system in advance.
}

11 Thus some references are omitted. 
examples presented in the paper are ready for implementation in Java, for instance. Also hidden algebra fully inherits the power of abstract data type specification, by which one can define static, architectural aspects of software systems (by modules expressions) as well as basic data types within them.

Temporal logic enjoys the rigor of mathematical logics and has made considerable impact on formal verification through model checking technologies. We are afraid, however, that it may not support software design process (via refinement) very well since implementing temporal formulas (again for example in Java) can be very difficult. We are developing techniques for model checking behavioural specifications, which is briefly touched upon in Section 4 and planning to use temporal formulas as an input language.

I/O Automata [15] and evolving algebra [16] are probably the closest to the hidden algebra approach. This is not surprising since hidden algebra stems from early formalisation efforts for automata. We feel that these techniques are more or less compatible. However, hidden algebra has a clear relation with ordinary algebra via behavioural equivalence and can rely on equational reasoning with machine support systems such as CafeOBJ.

Coalgebra [13] stresses duality with algebra and has produced many nice theoretical (mostly category-theoretic) results including the construction of terminal coalgebra, Birkhoff like axiomatisability results, and so on. Hidden algebra (without non-behavioural operations) is actually a concrete case of coalgebra (e.g., 17]) just like many-sorted algebra is a concrete case of algebra (of endo-functors). Coalgebra provides strong supports for hidden algebra in the area of theoretical development.

We like to mention briefly a couple of related techniques that we think are effective if used with behavioural specification.

Abstract interpretation [14] can go beyond the limit of refinement-based verification as it goes in the other direction. However, giving a right abstraction can be very difficult. Combining these two (refinement and abstraction) seems very promising. Various safety properties can be proved in this way.

Model checking has become a major topic in automated verification because of the increased computational capability enabled by binary decision diagrams (BDD) techniques. Application is still limited to hardware since the BDD techniques only apply to finite state systems. It is now a common perception that model checking for software systems requires a combined use of deductive and model-theoretic techniques. We feel behavioural specification offers a very good common ground for this. The example in Section 4 is the first step toward this.

We are building an integrated specification/verification environment on top of the CafeOBJ system. The planned features include a more efficient built-in resolution engine, an inductive theorem prover, and a behavioural model checking system. We also plan to have more substantial examples of distributed algorithms, fault-tolerant protocols, and security protocols. This is very important not just for theoreticians to get feedback, but also for practitioners to enhance the applicability of the method. 
Acknowledgement. We thank Prof. Joseph Goguen for his pioneering work in algebraic specification theory and for creating a new exciting field of study for us.

\section{References}

[1] Goguen, J., Malcolm, G.: A Hidden Agenda. To appear in Theoretical Computer Science, also available as Technical Report CS97-538, Computer Sci.\& Eng. Dept., Univ. of Calif. at San Diego (1997)

[2] Futatsugi, K., Nakagawa, A.: An Overview of CAFE Specification Environment: an algebraic approach for creating, verifying, and maintaining formal specification over networks, Proc. of First IEEE Int'l. Conf. on Formal Engineering Methods (1997)

[3] Diaconescu, R., Futatsugi, K.: CafeOBJ Report. World Scientific (1998)

[4] Diaconescu, R: Behavioural Coherence in Object-oriented Algebraic Specification. Technical Report IS-RR-98-0017F, Japan Advanced Institute of Science and Technology (1998)

[5] Rosu, G., Goguen, J.: Hidden Congruent Deduction. To appear in Lecture Notes in Artificial Intelligence (1999)

[6] Bidoit, M., Hennicker, R., Wirsing, M.: Behavioural and Abstractor Specifications. Science of Computer Programming 25(2-3) (1995) 149-186

[7] Milner, R.: Communication and Concurrency. Prentice-Hall (1989)

[8] Bartlett, K., Scantlebury, R., Wilkinson, P.: A Note on Reliable Full-duplex Transmission over Half-duplex Links. Communication of the ACM 12(5) (1969) 260-261

[9] Malcolm, G., Goguen, J.: Proving Correctness of Refinement and Implementation. Technical Monograph PRG-114, Programming Research Group, University of Oxford (1994)

[10] Goguen, J.. Mori, A., Lin, K, Rosus, G., Sato, A.: Distributed Cooperative Formal Methods Tools. Proc. of IEEE First Int'l Conf. on Automated Software Engineering (1997) 55-62

[11] Paramarcos, M., Patel, J.: A Low-Overhead Coherence Solution for Multiprocessors with Private Cache Memories. Proc. of Eleventh Int'l. Symp. on Computer Architecture (1984) 348-354

[12] Pong, F.: Symbolic State Model: A New Approach for the Verification of Cache Coherence Protocols. Ph.D. Thesis, Dept. of Electrical Engineering-Systems, Univ. of Southern California (1995)

[13] Jacobs, B.: Invariants, Bisimulations and the Correctness of Coalgebraic Refinements. Lecture Notes in Computer Science 1349 (1998) 276-291

[14] Cousot, P., Cousot, R.: Refining Model Checking by Abstract Interpretation. To appear in Automated Software Engineering Journal 6(1) (1999) 69-95

[15] Lynch, N.: Distributed Algorithms. Morgan Kaufman Pub. Inc. (1996)

[16] Gurevich, Y.: Evolving Algebras 1993: Lipari Guide. In Börger, ed. Specification and Validation Methods, Oxford University Press (1995) 9-36

[17] Cîrstea, C.: Coalgebra Semantics for Hidden Algebra: Parameterised Objects and Inheritance. Lecture Notes in Computer Science 1376 (1998) 


\section{A A CafeOBJ Session for the Alternating Bit Protocol}

mori (d194-054) [1024] cafeobj

-- loading standard prelude

Loading/usr/local/cafeobj-1.4/prelude/std.bin

Finished loading/usr/local/cafeobj-1.4/prelude/std.bin

-- CafeOBJ system Version 1.4.2(b3+) -built: 1998 Nov 28 Sat 6:29:00 GMT

prelude file: std.bin

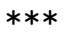

$1999 \mathrm{Feb} 10$ Mon 16:46:26 GMT

Type ? for help

uses GCL (GNU Common Lisp)

Licensed under GNU Public Library License

Contains Enhancements by W. Schelter

CafeOBJ> in abp

processing input : ./abp.mod

-- defining module! DATA_*.._* done.

-- defining module! QUEUE_*_*...........* done.

-- defining module* SENDER............*

** system already proved $=*=$ is a congruence of SENDER done.

-- defining module* RECEIVER............*

$* *$ system already proved $=*=$ is a congruence of RECEIVER done.

-- defining module* ABP, , , , , ,*_*............

** system failed to prove $=*=$ is a congruence of $\mathrm{ABP}$ done.

CafeOBJ> in test

processing input : ./test.mod

-- opening module ABP.. done.

-- reduce in \% : $\operatorname{Val}(\operatorname{Out}(\operatorname{In}(2, \operatorname{Out}(\operatorname{In}(1, \operatorname{Init})))))$

2 : NzNat

(0.000 sec for parse, 99 rewrites $(0.020 \mathrm{sec}), 142$ matches)

-- reduce in \% $\mathrm{Val}(\operatorname{Out}(\operatorname{In}(2, \operatorname{In}(1, \operatorname{In} i t))))$

1 : NzNat

( $0.000 \mathrm{sec}$ for parse, 46 rewrites $(0.000 \mathrm{sec}), 78$ matches $)$

-- reduce in \% : $\operatorname{Val}(\operatorname{Out}(\operatorname{In}(3, \operatorname{In}(2, \operatorname{In}(1, \operatorname{Init})))))$

1 : NzNat

( $0.000 \mathrm{sec}$ for parse, 53 rewrites $(0.010 \mathrm{sec}), 96$ matches)

-- reduce in \% $\operatorname{Val}(\operatorname{Out}(\operatorname{In}(3, \operatorname{In}(2, \operatorname{Out}(\operatorname{In}(1, \operatorname{Init}))))))$

2 : NzNat

(0.000 sec for parse, 114 rewrites $(0.010 \mathrm{sec}), 170$ matches)

-- reduce in \% : $\operatorname{Val}(\operatorname{Out}(\operatorname{In}(3, \operatorname{Out}(\operatorname{Out}(\operatorname{In}(2, \operatorname{Out}(\operatorname{In}(1, \operatorname{Init}))))))))$

3 : NzNat

(0.000 sec for parse, 250 rewrites $(0.020 \mathrm{sec}), 354$ matches)

CafeOBJ> 